\title{
EFICIENCIA Y EFECTIVIDAD DEL MÉTODO DE CONTINUACIÓN APLICADO AL DISEÑO BIAXIAL DE COLUMNAS CORTAS DE CONCRETO REFORZADO
}

\section{EFFICIENCY AND EFFECTIVENESS THE CONTINUATION METHOD APPLIED TO BIAXIAL DESIGN OF REINFORCED CONCRETE SHORT COLUMNS}

\author{
\begin{tabular}{l|l} 
Álvaro Ortega-Sierra & \\
& Breiner Reynaldo Sierra-Santos
\end{tabular}
}

Forma de citar: SIERRA-ORTEGA Alvaro, SIERRA-SANTOS Breiner. Eficiencia y efectividad del método de continuación aplicado al diseño biaxial de columnas cortas de concreto reforzado. Respuestas. 2013; 18(2): 6-15.

Recibido:

Febrero 20 de 2013

Aceptado:

Agosto 10 de 2013

\section{RESUMEN}

La investigación se realizó con 178 diseños correspondientes a columnas de sección cuadrada, por medio de un estudio descriptivo y cuasi experimental. Para dar solución al sistema de tres ecuaciones no lineales que gobierna el diseño se aplicó el Método de Continuación. De acuerdo con éste método, una solución equivalente a la del sistema anterior está dada por la que se obtiene cuando $t=1$ en un problema de valor inicial en el intervalo $0 \leq t \leq 1$, el cual fue resuelto implementando el método de Runge-Kutta de orden 4 con diferentes tamaños de paso. Para explorar la eficiencia se investigó acerca de la existencia de diferencias significativas entre promedios del error numérico al final de dos iteraciones consecutivas, considerando un máximo de cinco. Respecto a la efectividad, ésta se cuantificó para diferentes tolerancias del error en función del tamaño de paso y del número de iteraciones..

Palabras clave: flexión biaxial, eficiencia, efectividad, problema de valor inicial, Método de Runge-Kutta, tamaño de paso, iteración.

\section{ABSTRACT}

The research was conducted with 178 designs for square columns, using a quasi-experimental descriptive study. To solve the system of three nonlinear equations that govern the design was applied continuation method. According to this method, an equivalent solution to the above system is given by the one obtained when an initial value problem in the interval $0 \leq t \leq 1$, which was resolved by implementing the Runge-Kutta method of order 4 with different sizes step. To explore the efficiency was investigated on the existence of significant differences between means of numerical error at the end of two consecutive iterations, considering a maximum of five. With regard to effectiveness, this was quantified for different tolerances of the error depending on the step size and number of iterations.

Keywords: biaxial bending, efficiency, effectiveness, initial value problem, Runge-Kutta method, step size, iteration. 


\section{INTRODUCCIÓN}

$\bar{G}$ problema de diseñar una columna corta de concreto reforzado en flexión biaxial de forma "exacta", implica resolver un sistema de tres ecuaciones no lineales que corresponden al equilibrio de fuerzas internas y compatibilidad de deformaciones en una sección transversal de la misma3. Con tal propósito, se recurrió al Método de Continuación propuesto por Davidenko en 1950. La bondad de éste método es que partiendo de una situación físicamente real se asegura la convergencia de forma continua, independientemente de la elección que se defina como aproximación inicial a la solución del sistema ${ }^{4}$, siempre que se verifiquen las hipótesis establecidas en el teorema de convergencia demostrado por Ortega y Rheinboldt en 1970s. Por lo anterior, recientemente investigadores y estudiantes de postgrado en sus tesis han hecho uso de este método en diversos contextos como electricidad ${ }^{6}$, termodinámica ${ }^{7}$, y química $^{8}$, entre otros. Se pretendió entonces con la presente investigación, ampliar el espectro de las aplicaciones en ingeniería, explorando su eficiencia y cuantificando su efectividad en función del número de iteraciones y del tamaño de paso cuando se aplica en el diseño biaxial de columnas cortas de sección transversal cuadrada. Otro de los objetivos de esta investigación consistió en verificar experimentalmente la bondad de convergencia que ofrece el método.

\section{MATERIALES Y MÉTODOS}

Ante la imposibilidad de controlar de forma absoluta por procedimientos estadísticos al azar las variables que intervienen en el diseño, como son carga excéntrica, dimensiones de la sección transversal, propiedades mecánicas del concreto y del acero de refuerzo, distribución del acero y cuantía admisible en la sección, la investigación se desarrolló a través de un estudio de tipo descriptivo y cuasi experimental definiendo una población de 178 diseños. Se consideraron secciones transversales cuadradas con lados entre $30 \mathrm{~cm}$ y $50 \mathrm{~cm}$ múltiplos de $10 \mathrm{~cm}$, cuantías de acero comprendidas entre el 0,1\% y el 7\% del área de la sección transversal, cargas verticales comprendidas entre 35 ton y 220 ton $(350$ $\mathrm{kN}$ y $2.200 \mathrm{kN}$ ) con excentricidades entre $5 \mathrm{~cm}$ y $40 \mathrm{~cm}$ múltiplos de $5 \mathrm{~cm}$, refuerzo simétrico respecto a los ejes centroidales de la sección transversal, con 4 filas de refuerzo en cada cara para secciones de $30 \mathrm{~cm} \times 30 \mathrm{~cm}$ y 6 filas de refuerzo en cada cara para las secciones de $40 \mathrm{~cm} \times 40 \mathrm{~cm}$ y $50 \mathrm{~cm} \times 50 \mathrm{~cm}$. Lo anterior, teniendo en cuenta lo establecido en los artículos 7.6.3 y 10.9.2 de la norma ACI318S-08, en los que se estipulan una distancia libre entre barras no menor de 1.5 veces el diámetro de la barra, ni de $40 \mathrm{~mm}$, y no menos de 4 barras en columnas rectangulares con estribos. El listado detallado de la población objeto de estudio puede consultarse en el referente?

${ }^{3}$ PARK, R. y PAULAY, T. Estructuras de concreto reforzado. 1 ed. México: Limusa, 1983. p. 111, 113-114, 160-162, 174.

${ }^{4}$ SAINZ SAPERA, Luis. Estudio de la formulación y resolución del problema del flujo armónico de cargas. Trabajo de Grado. Ingeniero Industrial. Barcelona: Universidad Politécnica de Catalunya. Facultad de Ingeniería. Departamento de Ingeniería Industrial, 1995. p. 47.

${ }^{5}$ BURDEN, Richard L. y FAIRES, J. Douglas. Análisis numérico. 7 ed. México: Thomson Learning, 2002. p. 637.

${ }^{6}$ SAINZ SAPERA, Luis. Estudio de la formulación y resolución del problema del flujo armónico de cargas. Trabajo de Grado. Ingeniero Industrial. Barcelona: Universidad Politécnica de Catalunya. Facultad de Ingeniería. Departamento de Ingeniería Industrial, 1995.

${ }^{7}$ RODRÍGUEZ VALLEJO, Daniel Felipe; RESTREPO PLAZAS, Brenda Magaly y VELÁZQUEZ JIMÉNEZ, Jorge Alberto. Simulador dinámico de reactores flujo tapón. En: Revista Investigaciones Aplicadas. 2009. vol. 1, no. 5.

${ }^{8}$ BEREZOWSKI, Marek. The application of the parametric continuation method for determining steady state diagrams in chemical engineering [online]. En: Chemical Engineering Science. 2010. vol. 14 july 2010. no. 65, no. 1

${ }^{9}$ SIERRA SANTOS, Breiner Reynaldo. Exploración y aplicación del Método de Homotopía o Continuación en el diseño biaxial de columnas cortas de concreto reforzado de sección transversal cuadrada. Trabajo de Grado. Ingeniero Civil. San José de Cúcuta: Universidad Francisco de Paula Santander. Facultad de Ingeniería. Departamento de Ingeniería Civil, 2012. p. 49-52. 
No. 2

Junio - Diciembre 2013 ISSN 0122-820X

PP: 6-15

\subsection{MÉTODO DE RESISTENCIA ULTIMA}

De acuerdo con éste método, las cargas de diseño resultan de amplificar las cargas de servicio con un factor de seguridad y son equivalentes a la capacidad de carga nominal en el elemento estructural afectada por un factor de reducción de capacidad $\phi$. En esta investigación se consideró $\phi=0,7$.

\subsubsection{Suposiciones y parámetros de diseño.} $\mathrm{Se}$ asumieron las siguientes hipótesis y parámetros de diseño:

- En la zona elástica del acero se aplicó la Ley de Hooke, esto es $f_{s}=\varepsilon_{s} E_{s}$, donde:

$f_{s}=$ valor del esfuerzo en el acero

$\varepsilon_{s}=$ deformación unitaria en el acero

$E_{s}=$ valor del módulo de elasticidad del acero de refuerzo.

$$
\begin{aligned}
E_{s}= & 2^{\prime} 000.000 \mathrm{kgf} / \mathrm{cm}^{2} \text { (200.000 Mpa), } \\
& \text { según lo estipula el Reglamento } \\
& \text { NSR-10 art.C.8.5.2 }
\end{aligned}
$$

- Se descontó del bloque de concreto en compresión el desalojado por el acero de refuerzo.

- $\varepsilon_{c}=$ Valor máximo de la deformación unitaria en la fibra extrema a compresión del concreto. $\varepsilon_{c}=0,003$, según el Reglamento NSR-10 art.C.10.2.3"

- $f_{c}^{\prime}=$ Valor de la resistencia a la compresión del concreto. $f_{c}^{\prime}=210 \mathrm{kgf} / \mathrm{cm}^{2}(21 \mathrm{MPa})$.

- $f_{y}=$ Valor del límite de fluencia en el acero. $f_{y}=4200 \mathrm{kgf} / \mathrm{cm}^{2}(420 \mathrm{MPa})$.

- Para $\varepsilon_{s}<\frac{f_{y}^{y}}{E_{s}}, f_{s}=\varepsilon_{s} E_{s}$, de lo contrario $f_{s}=f_{y}$, como dice el Reglamento
NSR-10 art.C.10.2.4

- Se despreció la resistencia a la tracción del concreto, según dice el Reglamento NSR10 art.C.10.2. $5^{13}$.

- Se asumió un esfuerzo de compresión en el concreto de $0,85 f_{c}^{\prime}$, distribuido uniformemente sobre una zona equivalente de compresión, limitada por los bordes de la sección transversal y por una línea recta paralela al eje neutro, en una distancia $a=c \beta_{1}$ de la fibra de máxima deformación sometida a compresión, como estipula el Reglamento NSR-10 art.C.10.2.7.1 ${ }^{14}$, donde:

$c=$ distancia desde la fibra de máxima deformación hasta el eje neutro, medida en una dirección perpendicular a dicho eje, según el Reglamento NSR-10 art.C.10.2.7.215.

$\beta_{1}=0,85$, teniendo en cuenta que $170 \leq f_{c}^{\prime} \leq 280 \mathrm{kgf} / \mathrm{cm}^{2}$

$\left(17 \leq f_{c}^{\prime} \leq 28 \mathrm{MPa}\right)$, según dice el Reglamento NSR-10 art.C.10.2.7.316.

2.1.2 Ecuación del eje neutro. En una sección transversal con carga axial y flexión biaxial, el eje neutro o la línea de la sección transversal donde los esfuerzos son nulos está inclinado respecto a la horizontal ${ }^{17}$. Respecto al sistema coordenado xy que se muestra en la figura 1 , si $\left(k_{x} b, 0\right)$ y $\left(0, k_{y} h\right)$ son las coordenadas de los puntos donde el eje neutro intercepta los ejes $\mathrm{x}$ e y, respectivamente, el eje neutro tiene por ecuación $\quad k_{y} h$, donde:

- $k_{x}$ y $y_{y}^{=}, k_{y} h_{\text {son }} \frac{k_{y} h}{d}$ os parámetros que definen la posiciốn del eje neutro.

- $b=$ ancho de la sección transversal.

- $h=$ altura de la sección transversal.

\footnotetext{
${ }^{10}$ ASOCIACIÓN COLOMBIANA DE INGENIERÍA SÍSMICA - AIS. Ley 400 de 1997. Decreto 926 del 19 de marzo de 2010-Reglamento colombiano de construcción sismo resistente-NSR-10. Tomo I. 1 ed. Bogotá: 3R Editores, 2010. p. C-113.

${ }^{11}$ Ibíd., p. C-137.

${ }^{12}$ Ibíd., p. C-137.

${ }^{13}$ Ibíd., p. C-138.

${ }^{14}$ Ibíd., p. C-138.

${ }^{15}$ Ibíd., p. C-139.

${ }^{16}$ Ibíd., p. C-139.

${ }^{17}$ PARK y PAULAY. Op. cit., p. 111.
} 
2.1.3 Deformaciones, esfuerzos y fuerzas en el acero. Con relación a la figura 1 , si $\left(x_{i}, y_{i}\right)$ son las coordenadas de una barra de refuerzo cualquiera $\mathbf{i}$, su desviación respecto al eje neutro es $z_{i}=k_{y} h-\frac{k_{y} h}{k_{x} b} x_{i}-y_{i}$

y su deformación unitaria $\varepsilon_{s i}$, considerando los triángulos semejantes del diagrama de deformación es $\varepsilon_{s i}=\frac{\varepsilon_{c} z_{i}}{k_{y} h}$. Entonces, para $\varepsilon_{c}=0,003$, resulta $\varepsilon_{s i}=\frac{0,003 z_{i}}{k_{y} h}$ y el respectivo esfuerzo $f_{s i}$, en la región lineal de la curva esfuerzo-deformación del acero está dado por $f_{s i}=\varepsilon_{s i} E_{s}=\frac{0,003 z_{i}}{k_{v} h} E_{s}$. En éste análisis, se consideraron positivos los esfuerzos y fuerzas de compresión. Además, para esfuerzos de compresión $f_{s i}>f_{v} \Rightarrow f_{s i}=f_{v}$, mientras que $f_{s i}<-f_{y} \Rightarrow f_{s i}=-f_{y}$ para esfuerzos de tensión.
Sean:

- $A_{s t}=$ Área total de acero en la sección

- $\rho=$ Cuantía de acero en proporción al área de la sección transversal

- $A_{s i}=$ Área de acero de la i-ésima barra

- $\lambda_{i}=$ Proporción entre el área de acero de la i-ésima barra y el área total de acero en la sección

- $S_{i}=$ Fuerza en la i-ésima barra de acero.

Entonces, para una sección rectangular de dimensiones $b$ y $h, A_{s t}=\rho b h, A_{s i}=\lambda_{i} A_{s t}=\lambda_{i} \rho b h$ y $S_{i}=A_{s i} f_{s i}=\lambda_{i} \rho b h f_{s i}$.

2.1.4 Fuerza de compresión en el concreto $\left(\mathrm{C}_{\mathrm{c}}\right)$. Esta fuerza y su ubicación dependen del perfil y del área del bloque de esfuerzo de compresión equivalente. Las cuatro áreas

Figura 1. Sección de columna con flexión biaxial en la carga última

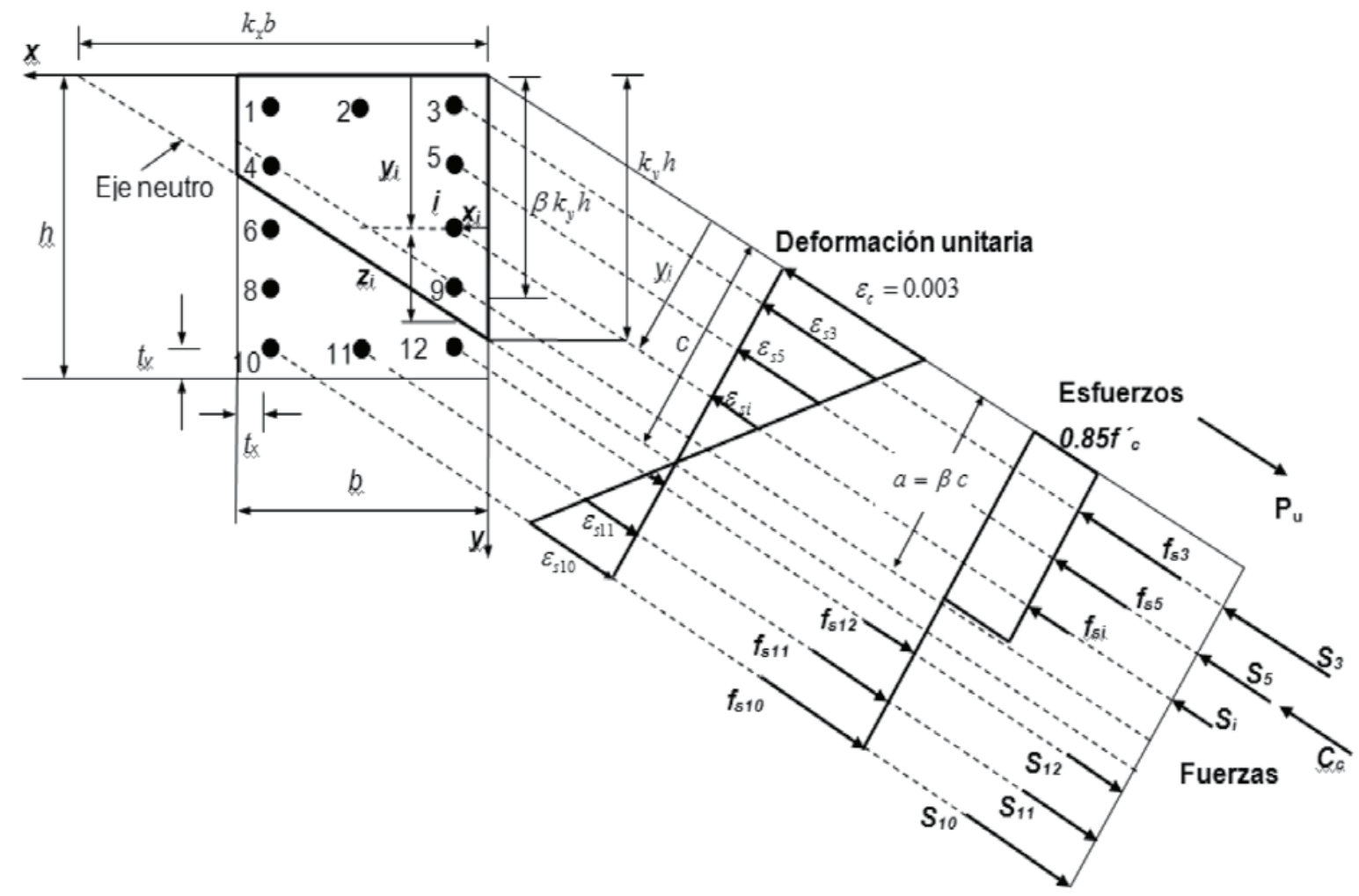

Fuente: PARK, R. y PAULAY, T. Estructuras de concreto reforzado. 1 ed. México: Limusa, 1983. Modificación autor. p. 162. 
No. 2

Junio - Diciembre 2013 ISSN 0122-820X

PP: 6-15 posibles son las que se muestran en la figura 2 . En cualquiera de éstos casos, $C_{c}=0,85 f_{c}^{\prime} A$ y se localiza en el centroide geométrico $(\bar{x}, \bar{y})$ del área $A$ del bloque de compresión. Tanto $A$, como $(\bar{x}, \bar{y})$, en cada caso, puede consultarse en el referente ${ }^{18}$.

2.1.5 Diseño. El diseño implica resolver tres ecuaciones no lineales que corresponden al equilibrio de fuerzas internas y compatibilidad de deformaciones en una sección transversal $^{19}$. Estas últimas involucran las variables $k_{x}, k_{y}, \rho$, que definen la fuerza de compresión en el concreto, las deformaciones unitarias, esfuerzos y fuerzas en el acero. Para la columna que se muestra en la figura 3, éste sistema es:

$$
\begin{array}{r}
f_{1}\left(k_{x}, k_{y}, \rho\right)=\left[C_{c}+\sum_{i=1}^{n} S_{i}\right]-\frac{P_{u}}{\phi}=0 \\
f_{2}\left(k_{x}, k_{y}, \rho\right)=\left[C_{c}\left(\frac{h}{2}-\bar{y}\right)\right]+\sum_{i=1}^{n} S_{i}\left(\frac{h}{2}-y_{i}\right)-\frac{M_{u x}}{\phi}=0 \\
f_{3}\left(k_{x}, k_{y}, \rho\right)=\left[C_{c}\left(\frac{b}{2}-\bar{x}\right)\right]+\sum_{i=1}^{n} S_{i}\left(\frac{b}{2}-x_{i}\right)-\frac{M_{u y}}{\phi}=0
\end{array}
$$

donde:

$$
\begin{aligned}
P_{u}= & \text { Carga de diseño } \\
e_{x}= & \text { Excentricidad de } P_{u}, \text { medida sobre } \\
& \text { la perpendicular al eje } Y_{G} \\
e_{y}= & \text { Excentricidad de } P_{u}, \text { medida sobre } \\
& \text { la perpendicular al eje } X_{G}
\end{aligned}
$$

Figura 2. Posibles áreas del bloque de compresión del concreto

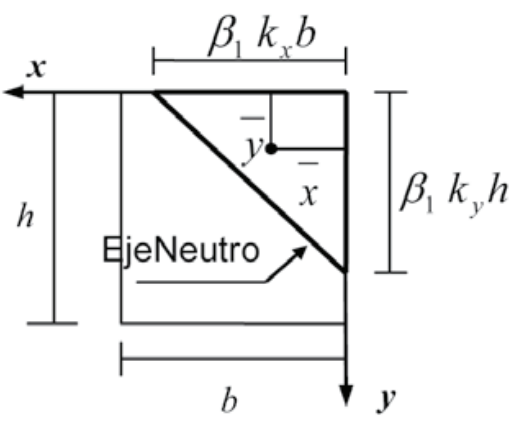

Caso 1. $\beta_{l} k_{y} h<h$ y $\beta_{l} k_{x} b<b$

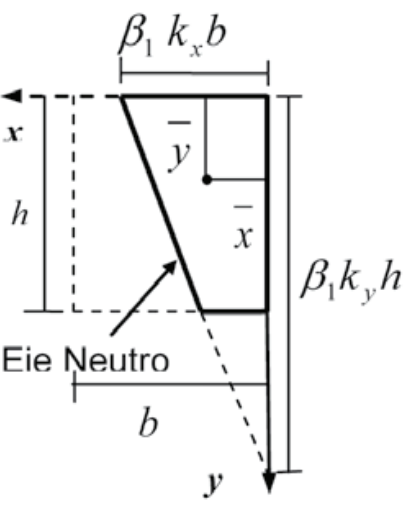

Caso 3. $\beta_{l} k_{y} h>h$ y $\beta_{l} k_{x} b<b$

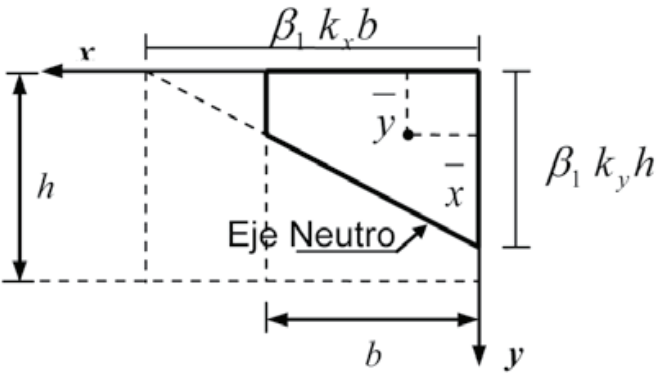

Caso 2. $\beta_{l} k_{y} h<h$ y $\beta_{l} k_{x} b>b$

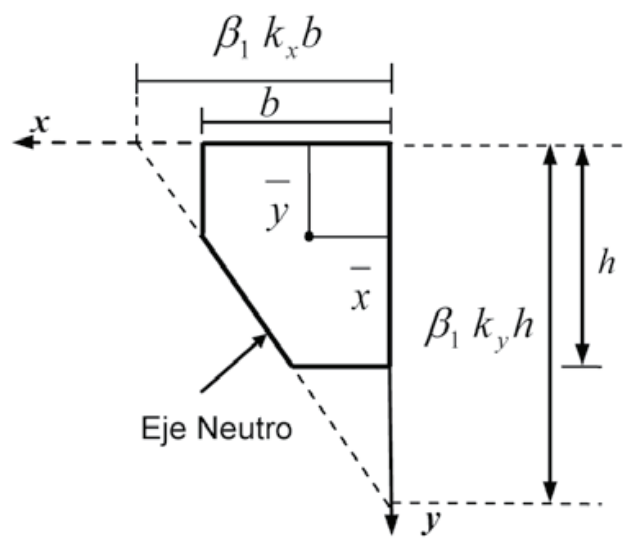

Caso 4. $\beta_{l} k_{y} h>h$ y $\beta_{l} k_{x} b>b$

Fuente: PARK, R. y PAULAY, T. Estructuras de concreto reforzado. 1 ed. México: Limusa, 1983. Modificación autor. p. 114.

${ }^{18}$ SIERRA SANTOS, Breiner Reynaldo. Op. cit., p. 35-37.

${ }^{19}$ PARK y PAULAY. Op. cit., p. 114 y 161. 

$\begin{aligned} M_{u X}= & P_{u} e_{y}=\text { Momento de diseño alre- } \\ & \text { dedor del eje } X_{G}\end{aligned}$

$M_{u Y}=P_{u} e_{x}=$ Momento de diseño alrededor del eje $Y_{G}$

Figura 3. Columna en flexión biaxial

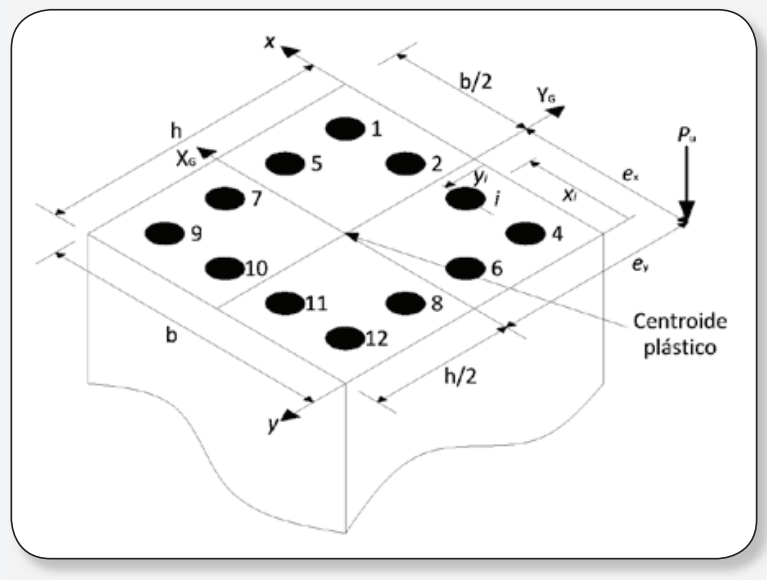

Fuente: PARK, R. y PAULAY, T. Estructuras de concreto reforzado. 1 ed. México: Limusa, 1983. Modificación autor. p. 161.

\subsection{MÉTODO DE HOMOTOPÍA O CONTINUACIÓN}

Para abreviar el sistema de ecuaciones (1), (2) y (3), sea:

(4) $[F(X)]=\left[\begin{array}{l}f_{1}\left(k_{x}, k_{y}, \rho\right) \\ f_{1}\left(k_{x}, k_{y}, \rho\right) \\ f_{1}\left(k_{x}, k_{y}, \rho\right)\end{array}\right]=\left[\begin{array}{l}0 \\ 0 \\ 0\end{array}\right]$

donde $X=\left(k_{x}, k_{y}, \rho\right)^{T}$. De acuerdo con éste método ${ }^{20}$, el vector solución $X^{*}$ del sistema (4) está dado por la solución en $t=1 \quad\left(X^{*}=X(1)\right)$ del problema de valor inicial conformado por el sistema de ecuaciones diferenciales $\left(\frac{d}{d t}(X(t))\right)=-[J(X(t))]^{-1}[F(X(0))]$ en $0 \leq t \leq 1$, donde $X(0)=\left(k_{x}(0), k_{y}(0), \rho(0)\right)^{T}$ representa la condición inicial y $[J(X(t)]$ la matriz Jacobiana de las funciones $[F(X)]$.
Para aproximar las derivadas presentes en la matriz Jacobiana en el intervalo $0 \leq t<1$ se utilizaron diferencias finitas centrales ${ }^{21}$, definiendo incrementos $\Delta k_{x}=\Delta k_{y}=\Delta \rho=0,0001$

En la aproximación de la solución $X(1)=\left(k_{x}(1), k_{y}(1), \rho(1)\right)^{T}$ del sistema de ecuaciones diferenciales se usó el Método de Rungekutta de orden 4, implementando el Método de Continuación de forma iterativa, considerando un máximo de 5 . En la primera iteración se definió de forma a priori la condición inicial $\quad X^{(1)}(0)=\left(k_{x}(0), k_{y}(0), \rho(0)\right)^{T}=(0.5,0.5,0.01)^{T}$ que corresponden al caso 1 de las posibles formas del bloque de compresión del concreto ilustrado en la figura 2 y una cuantía mínima de diseño $\rho=0,001$, la cual representa una situación física real, mientras que para la iteración i+1 se consideró $X^{(i+1)}(0)=X^{(i)}(1)$.

\subsection{EFICIENCIA Y EFECTIVIDAD DEL MÉTODO DE CONTINUACIÓN}

2.3.1 Error numérico $\varepsilon^{(i)}(\Delta t)$ al final de la i-ésima iteración. Éste se definió en función del tamaño de paso $\Delta t$ considerado al usar el Método de RungeKutta de orden 4 y del vector de residuos $\left[\varepsilon_{1}^{(i)}, \varepsilon_{2}^{(i)}, \varepsilon_{3}^{(i)}\right]^{T}=\left[f_{1}^{(i)}(X(1)), f_{2}^{(i)}(X(1)), f_{3}^{(i)}(X(1))\right]^{T}$ en las ecuaciones de equilibrio al final de la i-ésima iteración, a través de la expresión:

(5) $\varepsilon^{(i)}(\Delta t)=f\left(\varepsilon_{1}^{(i)}, \varepsilon_{2}^{(i)}, \varepsilon_{3}^{(i)}\right)=\sqrt{\left(\varepsilon_{1}^{(i)}\right)^{2}+\left(\varepsilon_{2}^{(i)}\right)^{2}+\left(\varepsilon_{3}^{(i)}\right)^{2}}$

2.3.2. Instrumento de Cómputo. Consistió en la elaboración de una hoja de cálculo con una macro en Visual Basic para Excel. Ésta arroja como resultados la aproximación numérica a la solución del sistema $F(X)=0$ y el vector de residuos $\left(\varepsilon_{1}^{(i)}, \varepsilon_{2}^{(i)}, \varepsilon_{3}^{(i)}\right)^{T}{ }_{22}$.

Junio - Diciembre 2013 ISSN 0122-820X

PP: 6-15
2.3.3 Tamaño de paso óptimo con una iteración. Después de aplicar el instrumento a cada uno de los 178 diseños considerados, con tamaños de paso $\Delta t=1 / N$, para $N=2,3,4$,

${ }^{20}$ BURDEN y FAIRES. Op. cit., p. 635-636.

${ }^{21}$ NIEVES HURTADO, Antonio y DOMÍNGUEZ SÁNCHEZ, Federico C. Métodos numéricos aplicados a la ingeniería. 2 ed. México: CECSA, 2003. p. 533.

${ }^{22}$ SIERRA SANTOS, Breiner Reynaldo. Op. cit., p. 52-53. 
No. 2

Junio - Diciembre 2013 ISSN 0122-820X

PP: 6-15

$5,6,7,8,9,10,20$, se determinó la media poblacional del error numérico correspondiente en función de $\Delta t$ mediante la expresión:

$$
\mu_{1}(\Delta t)=\frac{\sum_{i=1}^{i=178} \varepsilon^{(1)}(\Delta t)}{178}=\frac{\sum_{i=1}^{i=178} \sqrt{\left(\varepsilon_{1}^{(1)}\right)^{2}+\left(\varepsilon_{2}^{(1)}\right)^{2}+\left(\varepsilon_{3}^{(1)}\right)^{2}}}{178}
$$

Teniendo en cuenta que el tamaño de paso $\Delta t$ es una variable aleatoria discreta, el tamaño de paso óptimo $\Delta t$ con una iteración se estableció igual al valor de $\Delta t$ que generó la menor media poblacional $\mu_{1}$.

2.3.4 Efectividad. Ésta se cuantificó definiendo $\Delta t=\Delta t_{o p}$, teniendo en cuenta el número de iteraciones y valores de tolerancia en el error numérico. Entonces, si:

$\varepsilon_{t}=$ tolerancia admisible para el error numérico $\varepsilon^{(i)}(\Delta t)$

$n_{i}=$ frecuencia de ocurrencia de diseños en los que se observó $\varepsilon^{(i)}(\Delta t) \leq \varepsilon_{t}$, la efectividad del método para la población en estudio al final de la i-ésima iteración está dada por $E_{f i}=\frac{n_{i}}{178} \times 100 \%$.

2.3.5 Eficiencia. Para explorar la eficiencia se investigó acerca de la existencia de diferencias significativas entre promedios del error numérico al final de dos iteraciones consecutivas con $\Delta t=\Delta t_{o p}$ y considerando un máximo de cinco, realizando pruebas de comparación de medias para poblaciones pareadas con una significancia $\alpha=0,05$.

\section{Resultados y discusión}

Los resultados que se dan a conocer a continuación y las conclusiones que de ellos se derivan sólo son válidos para la población de diseños considerados, correspondientes a columnas cortas con la carga aplicada sobre la diagonal de la sección cuadrada

Respecto al vector de residuos $\left[\varepsilon_{1}^{(i)}, \varepsilon_{2}{ }^{(i)}, \varepsilon_{1}^{(i)}\right]^{T}=\left[f_{1}^{(i)}(X(1)), f_{2}^{(i)}(X(1)), f_{3}^{(i)}(X(1))\right]^{T}$ en las ecuaciones de equilibrio al final de la i-ésima que definen el error numérico en la ecuación (5), estos pueden consultarse en el Anexo J de la fuente referenciada en la tabla 1. Las unidades del error numérico pueden asimilarse en toneladas $(1$ ton $=10 \mathrm{kN})$, teniendo en cuenta que la mayor contribución al error la aporta el residuo $\varepsilon_{1}^{(i)}=f_{1}^{(i)}(X(1))$, correspondiente al equilibrio de fuerzas en la dirección longitudinal de la columna

\subsection{ERROR NUMÉRICO MEDIO AL FINAL DE LA PRIMERA ITERACIÓN}

Nótese en la tabla 1 que al reducir $\Delta t$ desde $1 / 2$ hasta $1 / 7$ el error numérico medio $\mu_{1}(\Delta t)$ es decreciente, excepto de $1 / 5$ a $1 / 6$, mientras que si $\Delta t<1 / 7$ éste tiende a incrementase, por lo que $\Delta t_{o p}=1 / 7$. Lo anterior puede explicarse debido que a menor tamaño de paso se requieren más cálculos y en consecuencia es predecible un mayor error de redondeo.

Tabla 1. Error

Numérico medio con una iteración

\begin{tabular}{|cc|}
\hline$\Delta t$ & $\mu_{1}(\Delta t)$ \\
\hline $1 / 20$ & 2,02 \\
$1 / 10$ & 2,13 \\
$1 / 9$ & 2,12 \\
$1 / 8$ & 2,01 \\
$1 / 7$ & 1,90 \\
$1 / 6$ & 2,13 \\
$1 / 5$ & 2,12 \\
$1 / 4$ & 2,46 \\
$1 / 3$ & 2,88 \\
$1 / 2$ & 8,96 \\
\hline
\end{tabular}

Fuente: SIERRA SANTOS, Breiner Reynaldo. Exploración y aplicación del Método de Homotopía o Continuación en el diseño biaxial de columnas cortas de concreto reforzado de sección transversal cuadrada. Trabajo de Grado. Ingeniero Civil. San José de Cúcuta: Universidad Francisco de Paula Santander. Facultad de Ingeniería. Departamento de Ingeniería Civil, 2012. p. 58. 
Tabla 2. Efectividad $E_{f i}$ o frecuencia $n_{i}$ con que $\varepsilon^{(i)} \leq \varepsilon_{t}$

\begin{tabular}{|ccccccccc|}
\hline \multicolumn{10}{|c|}{ TOLERANCIAS DEL ERROR $\left(\varepsilon_{t}\right)$} \\
\hline \multicolumn{10}{|c}{$\varepsilon_{t}=0,05$} & \multicolumn{1}{c|}{$\varepsilon_{t}=0,02$} & $\varepsilon_{t}=0,01$ & \multicolumn{2}{c|}{$\varepsilon_{t}=0,001$} \\
\hline ITER & Frec. & $E_{f i}$ & Frec. & $E_{f i}$ & Frec. & $E_{f i}$ & Frec. & $E_{f i}$ \\
(i) & $\left(n_{i}\right)$ & $\mathbf{( \% )}$ & $\left(n_{i}\right)$ & $(\%)$ & $\left(n_{i}\right)$ & $(\%)$ & $\left(n_{i}\right)$ & $(\%)$ \\
1 & 1 & 0,56 & 0 & 0 & 0 & 0 & 0 & 0 \\
2 & 173 & 97,19 & 173 & 97,19 & 173 & 97,19 & 169 & 94,94 \\
3 & 177 & 99,44 & 177 & 99,44 & 177 & 99,44 & 177 & 99,44 \\
4 & 177 & 99,44 & 177 & 99,44 & 177 & 99,44 & 177 & 99,44 \\
5 & 177 & 99,44 & 177 & 99,44 & 177 & 99,44 & 177 & 99,44 \\
\hline
\end{tabular}

Junio - Diciembre 2013

ISSN 0122-820X

Fuente: SIERRA SANTOS, Breiner Reynaldo. Exploración y aplicación del Método de Homotopía o Continuación en el diseño biaxial de columnas cortas de concreto reforzado de sección transversal cuadrada. Trabajo de Grado. Ingeniero Civil. San José de Cúcuta: Universidad Francisco de Paula Santander. Facultad de Ingeniería. Departamento de Ingeniería Civil, 2012. p. 60.

\subsection{EFECTIVIDAD DEL MÉTODO}

El valor de la efectividad $E_{f i}$ al final de cada una de las cinco primeras iteraciones, con un tamaño de paso $\Delta t$ igual al óptimo observado al final de la primera iteración $\left(\Delta t_{o p}=1 / 7\right)$, para diferentes valores de tolerancia $\varepsilon_{t}$ se da a conocer en la tabla 2. Nótese que la efectividad del método es directamente proporcional al número de iteraciones. Además, para una tolerancia del error $\varepsilon_{t}=0.001$, la efectividad observada $E_{f}$ es del $99,44 \%$ al final de la tercera, cuarta y quinta iteración, serían entonces suficientes sólo 3 iteraciones para que el error numérico $\varepsilon^{(i)}$ quede acotado en el intervalo $0<\varepsilon^{(i)}(\Delta t)<0.001$ en 99 de 100 veces que se implemente el método con fines de diseño.

\subsection{EFICIENCIA}

Aunque la Efectividad del método es la misma al final de la tercera, cuarta y quinta iteración, estadísticamente pudieran existir o no diferencias significativas entre las medias poblacionales del error numérico $\mu_{i}$ y $\mu_{i+1}$ al final de dos iteraciones consecutivas i e $i+1$. Por lo anterior, con el objeto de recomendar una implementación eficiente del método, esto es, no iterarlo infructuosamente más de lo debido, se verificó estadísticamente mediante una prueba para poblaciones relacionadas en que iteraciones consecutivas no se presentan diferencias significativas entre las medias poblacionales del error

3.3.1 Pruebas de bondad de ajuste a la normal. Se realizaron definiendo un nivel de significancia $\alpha=0,05$. El estadístico Z(K-S) de Kolmogorov-Smirnov y los valores $p_{i, i+1}$ de significancia observada para cada una de las poblaciones de diferencias $\left[\varepsilon^{(i)}-\varepsilon^{(i+1)}\right]$ del error numérico, al final de dos iteraciones consecutivas i e $i+1$, se dan a conocer en la tabla 3. Puesto que siempre $p_{i, i+1}<\alpha$, ninguna de estas poblaciones sigue una distribución normal. Por lo anterior, se recurrió a la prueba no paramétrica de Wilcoxon para poblaciones relacionadas con el objeto de determinar si las medias poblacionales del error numérico difieren en dos iteraciones consecutivas.

3.3.2 Pruebas de Wilcoxon para poblaciones relacionadas. Se consideraron las hipótesis $H_{0}: \mu_{i+1}=\mu_{i}$ y $H_{a}: \mu_{i+1} \neq \mu_{i}$, donde $\mu_{i}$ y $\mu_{i+1}$ representan las medias poblacionales de los errores numéricos al final de las iteraciones i e $\mathrm{i}+1$. Los resultados de las pruebas, con un nivel de significancia $\alpha=0,05$, se dan a conocer en la tabla 4. 
Los valores $p_{i, i+1}$ de significancia observada indican que existen diferencias significativas entre las medias poblacionales de los errores al final de dos iteraciones consecutivas, hasta la cuarta $\left(p_{i, i+1}<0,05\right)$, mientras que los errores al final de la quinta ya no difieren de los de la precedente.

Tabla 3. Pruebas de bondad de ajuste

\begin{tabular}{|lcc|}
\hline$\left[\varepsilon^{(i)}-\varepsilon^{(i+1)}\right]$ & $Z(\mathrm{~K}-\mathrm{S})$ & $p_{i, i+1}$ \\
\hline$\left[\varepsilon^{(1)}-\varepsilon^{(2)}\right]$ & 1,762 & 0,004 \\
{$\left[\varepsilon^{(2)}-\varepsilon^{(3)}\right]$} & 6,906 & 0,000 \\
{$\left[\varepsilon^{(3)}-\varepsilon^{(4)}\right]$} & 6,994 & 0,000 \\
{$\left[\varepsilon^{(4)}-\varepsilon^{(5)}\right]$} & 6,994 & 0,000 \\
{$\left[\varepsilon^{(i)}-\varepsilon^{(i+1)}\right]$} & 6,994 & 0,000 \\
$\mathrm{Ho}:\left[\varepsilon^{(i)}-\varepsilon^{(i+1)}\right]$ tiene distribución normal \\
$\mathrm{Ha}:\left[\varepsilon^{(i)}-\varepsilon^{(i+1)}\right]$ no tiene distribución normal \\
\hline
\end{tabular}

Fuente: SIERRA SANTOS, Breiner Reynaldo. Exploración y aplicación del Método de Homotopía o Continuación en el diseño biaxial de columnas cortas de concreto reforzado de sección transversal cuadrada. Trabajo de Grado. Ingeniero Civil. San José de Cúcuta: Universidad Francisco de Paula Santander. Facultad de Ingeniería. Departamento de Ingeniería Civil, 2012. p. 71.

Tabla 4. Pruebas de Wilcoxon

\begin{tabular}{|ccccc|}
\hline \multicolumn{6}{|c|}{$\mu_{i}$} & $\mu_{i+1}$ & $W$ & $p_{i, i+1}$ \\
\hline 1 & $1.90 \mathrm{E}-00$ & $1,91 \mathrm{E}-02$ & $-11,570$ & $5,82 \mathrm{E}-31$ \\
2 & $1,91 \mathrm{E}-02$ & $1,79 \mathrm{E}-03$ & $-11,321$ & $1,04 \mathrm{E}-29$ \\
3 & $1,79 \mathrm{E}-03$ & $4,23 \mathrm{E}-04$ & $-2,625$ & $8,66 \mathrm{E}-03$ \\
4 & $4,23 \mathrm{E}-04$ & $4,72 \mathrm{E}-04$ & $-0,501$ & 0,617 \\
\hline
\end{tabular}

Fuente: SIERRA SANTOS, Breiner Reynaldo. Exploración y aplicación del Método de Homotopía o Continuación en el diseño biaxial de columnas cortas de concreto reforzado de sección transversal cuadrada. Trabajo de Grado. Ingeniero Civil. San José de Cúcuta: Universidad Francisco de Paula Santander. Facultad de Ingeniería. Departamento de Ingeniería Civil, 2012. p. 72.

\section{CONCLUSIONES Y RECOMENDACIONES}

Independientemente del valor de $\Delta t$, al final de la primera iteración el error numérico medio no tiende a cero, el tamaño de paso óptimo observado fue $\Delta t_{o p}=1 / 7$. Sin embargo, para éste valor de $\Delta t$, el vector $X^{(1)}(1)$ obtenido al final de la primera iteración constituye una excelente condición inicial $X^{(2)}(0)$ para la segunda, el error numérico al final de ésta última se reduce a 0,0191.

Como era de esperarse, la efectividad del Método de Continuación cuando se define un tamaño de paso $\Delta t=1 / 7$ es directamente proporcional al número de iteraciones, pero deja de ser eficiente con cinco o más, ya que no se apreciaron diferencias significativas en el error numérico al final de la cuarta y quinta iteración. Por lo anterior, no se recomienda realizar más de cuatro iteraciones.

La efectividad del método con un tamaño de paso $\Delta t=1 / 7$ es la misma al final de la tercera, cuarta y quinta iteración, aun para la tolerancia más exigente $\varepsilon_{t}=0,001$, la probabilidad de que el error numérico sea mayor a 0,001 es del 0,56\%, lo que hace al método confiable en un $99,44 \%$.

Aunque se verificó que el método tiene una alta efectividad y converge cuando se le implementa de forma recursiva, el trabajo numérico es dispendioso. Por ejemplo, cuando se resuelve un sistema de tres ecuaciones no lineales se requiere invertir cuatro matrices de $3 \times 3$ en cada paso para determinar doce constantes en el Método de Runge-Kutta de orden 4 . Un total de $4 \times 7 \times 3=84$ matrices por invertir cuando se define $\Delta t=1 / 7$ y se realizan tres iteraciones ${ }^{23}$.

Aunque se presentan diferencias significativas entre las medias del el error numérico al final de la tercera y cuarta iteración su efectividad se mantiene en un $99,44 \%$, por lo que se re-

\footnotetext{
${ }^{23}$ SIERRA SANTOS, Breiner Reynaldo. Op. cit., p. 59.
} 
comiendan tres iteraciones, al final de esta última $\mu_{3}=0,00179$.

Teniendo en cuenta que el Método de Continuación converge cuando se le implementa de forma iterativa y con el objeto de reducir el trabajo numérico, se propone explorar la eficiencia y efectividad del Método de Newton-Raphson en varias variables para resolver las ecuaciones de diseño, definiendo como aproximación inicial el vector $X^{(1)}(1)$, o bien, el vector $X^{(2)}(1)$.

Considerando que el método de NewtonRaphson es mucho más sencillo y que los cálculos numéricos son considerablemente menores comparados con los que exige el método de continuación, resultaría interesante explorar la eficiencia y efectividad del primero y compararlas con las que ofrece éste último.

Puesto que el Método de Continuación converge a la solución exacta, puede usarse como referente para cuantificar el error que presentan otros métodos de diseño tales como el método de superposición, el método de la excentricidad uniaxial equivalente, el método de la carga inversa, el método del contorno de carga y las gráficas de diseño de Row y Paulay, entre otros.

\section{REFERENCIAS}

ASOCIACIÓN COLOMBIANA DE INGENIERÍA SÍSMICA - AIS. Ley 400 de 1997. Decreto 926 del 19 de marzo de 2010-Reglamento colombiano de construcción sismo resistente-NSR-10. Tomo I. 1 ed. Bogotá: 3R Editores, 2010. 727 p.

BEREZOWSKI, Marek. The application of the parametric continuation method for determining steady state diagrams in chemical engineering [online]. En: Chemical Engineering Science. 2010. vol. 14 july 2010. no. 65, no. 1 , p. 5411-5414.

BURDEN, Richard L. y FAIRES, J. Douglas. Análisis numérico. 7 ed. México: Thomson
Learning, 2002.839 p.

NIEVES HURTADO, Antonio y DOMÍNGUEZ SÁNCHEZ, Federico C. Métodos numéricos aplicados a la ingeniería. 2 ed. México: CECSA, 2003. 600 p.

PARK, R. y PAULAY, T. Estructuras de concreto reforzado. 1 ed. México: Limusa, 1983. $796 \mathrm{p}$.

RODRÍGUEZ VALLEJO, Daniel Felipe; RESTREPO PLAZAS, Brenda Magaly y VELÁZQUEZ JIMÉNEZ, Jorge Alberto. Simulador dinámico de reactores flujo tapón. En: Revista Investigaciones Aplicadas. 2009. vol. 1, no. 5, p. 44-54.

SAINZ SAPERA, Luis. Estudio de la formulación y resolución del problema del flujo armónico de cargas. Trabajo de Grado. Ingeniero Industrial. Barcelona: Universidad Politécnica de Catalunya. Facultad de Ingeniería. Departamento de Ingeniería Industrial, 1995. 142 p.

SIERRA SANTOS, Breiner Reynaldo. Exploración y aplicación del Método de Homotopía o Continuación en el diseño biaxial de columnas cortas de concreto reforzado de sección transversal cuadrada. Trabajo de Grado. Ingeniero Civil. San José de Cúcuta: Universidad Francisco de Paula Santander. Facultad de Ingeniería. Departamento de Ingeniería Civil, 2012. 426 p.
Junio - Diciembre 2013

ISSN 0122-820X

PP: 6-15 